\title{
Influence of the immobilized subtilisins on perfomance indicators of the rat heart in experiment
}

\author{
German Baikalov \\ Department of experimental \\ pharamacology \\ Research Institute of Clinical and \\ Experimental Lymphology - Branch of the \\ Institute of Cytology and Genetics Siberian \\ Branch of the Russian Academy of \\ Sciences Novosibirsk \\ Novosibirsk, Russian Federation \\ Department of pharmacology, clinical \\ pharmacology and evidence based \\ medicine \\ Novosibirsk State Medical University \\ Novosibirsk, Russian Federation \\ gbaikalov@yandex.ru
}

\author{
Roman Knyazev \\ Research Institute of Biochemistry - \\ Branch of the Federal Research Center of \\ Fundamental and Translational Medicine \\ Department of pharmacology, clinical \\ pharmacology and evidence based \\ medicine \\ Novosibirsk State Medical University \\ Novosibirsk, Russian Federation \\ knjazev_roman@mail.ru \\ Pavel Madonov \\ Department of experimental \\ pharamacology \\ Research Institute of Clinical and \\ Experimental Lymphology - Branch of the \\ Institute of Cytology and Genetics Siberian \\ Branch of the Russian Academy of \\ Sciences \\ Novosibirsk, Russian Federation \\ Department of pharmacology, clinical \\ pharmacology and evidence-based \\ medicine \\ Novosibirsk State Medical University \\ Novosibirsk, Russian Federation \\ pmadonov@yandex.ru
}

\author{
Konstantin Ershov \\ Department of pharmacology, clinical \\ pharmacology and evidence-based \\ medicine \\ Novosibirsk State Medical University \\ Novosibirsk, Russian Federation \\ ershov_k@bk.ru
}

\begin{abstract}
The immobilized subtilisins are bacterial proteinases with a high thrombolytic activity, capable of improving peripheral blood circulation by dissolving the blood clot and reducing manifestations of endothelial dysfunction. This article presents the materials of the effect of immobilized subtilisins on performance heart indicators in the experiment. The action of these proteinases was studied on a model of isolated rat heart according to Langendorff. Indicators of heart rate (HR), pressure developed by the left ventricle (LVP), heart efficiency (HE) were selected for registration. It was shown when adding the immobilized subtilisins, an increase of the left ventricular contraction force and a decrease in a heart rate were observed. The results indicate that immobilized subtilisins have positive inotropic and negative chronotropic effects.
\end{abstract}

Keywords - immobilized subtilisins, isolated heart, heart rate, left ventricular pressure, heart efficiency

\section{Introduction}

Currently, the diseases of the circulatory system are an highly important problem and a cause of death worldwide. The largest percentage among them is coronary heart disease, including angina pectoris, myocardial infarction and sudden cardiac death. As a result of this, the search of effective pharmacological preparations for the correction and treatment of cardiovascular diseases is an urgent area.

The proteolytic enzymes have been used by humans for many centuries, but their scientifically reasoned use began in the early twentieth century after the publication of fundamental works by E. Fisher. The proteolytic enzymes can be obtained from plant materials (e.g., papain), from animal raw materials (e.g., trypsin), or from microorganisms. The subtilisins are proteolytic enzymes from the class of hydrolases, belong to the class of serine proteases and they are classified according to genera in accordance with the homology of the amino acid sequence. The name subtilisin comes from the fact that most of these enzymes are produced by the bacteria Bacillus subtilis. The possibility of the clinical use of subtilisins has been studied quite actively in recent years, and studies show their effectiveness not only for the treatment of thrombosis, but also for the prevention of cardiovascular diseases $[1,2]$.

There is no data in the literature about the effect of subtilisins on heart function. There are various models for experimental studies in the cardiology. One of the relevant models is a perfusion of isolated rat heart according to Langendorff. This model allows to explore and study in detail the physiological and biochemical processes in the heart as well as external influences on it in the absence of neurogenic and humoral factors [3-6].

The purpose of this work is to study the influence of immobilized subtilisins on the performance indicators of isolated rat heart according to Langendorff.

\section{Materials and Methods}

The experiments were performed on male Wistar rats weighing 250-300 g. An hour before the experiment, the animals were intraperitoneally injected with heparin (500 units per rat). After decapitation, the heart was quickly removed and placed in a container with perfusion solution at $\mathrm{t}=0{ }^{\circ} \mathrm{C}$. A cannula was inserted into the aorta and connected to a perfusion system. A perfusion was performed through the coronary vessels under a constant pressure of $70 \mathrm{mmHg}$. The 
modified Krebs-Henseleit buffer was used as a perfusate. The composition of the modified buffer solution Krebs - Henseleit is shown below (Table 1).

TABLE 1. THE COMPOSITION OF THE MODIFIED BUFFER SOLUTION KREBSHENSELEIT FOR PERFUSION OF ISOLATED RAT HEARTS

\begin{tabular}{|c|c|}
\hline Component & Concentration, mM \\
\hline $\mathrm{NaCl}$ & 118 \\
\hline $\mathrm{KCl}$ & 4,7 \\
\hline $\mathrm{CaCl}_{2}$ & 3,0 \\
\hline $\mathrm{MgSO}_{4}$ & 1,2 \\
\hline $\mathrm{KH}_{2} \mathrm{PO}_{4}$ & 1,2 \\
\hline $\mathrm{NaHCO}_{3}$ & 25 \\
\hline $\mathrm{EDTA}-\mathrm{Na}_{2}$ & 0,5 \\
\hline Glucose & 5 \\
\hline
\end{tabular}

We used a carbogen $(95 \% \mathrm{O} 2$ and $5 \% \mathrm{CO} 2)$ for heart saturation and conducted a constant monitoring of $\mathrm{pH}$ (7.4). The constant solution temperature was $37.5^{\circ} \mathrm{C}$. To register the pressure developed by the left ventricle, an incision was made in the left atrium behind the appendage, then a latex balloon was injected through it, subsequently filled with perfusion solution to the required volume and connected to a digital pressure sensor. The balloon was introduced first into the cavity of the atrium, and then through the mitral valve into the cavity of the left ventricle. The frequency and rate of contraction were recorded. The heart efficiency (HE) was defined as the product of the pressure increase to the frequency of contractions per minute.

The number of animals in the study group was 7. Each selected heart worked for at least 15 minutes without recirculating of the perfusion solution until the constant amplitude and frequency rates were established. Then, the investigated component was introduced into the perfusion solution, and the heart worked without recirculation for 40 minutes. The work presents data that is obtained at 5, 10, 20, 30,40 minutes of perfusion in the presence of the studied drug. The results will reflect the maximum values of performance indicators at the 20 minutes of perfusion. A record of the isolated heart working is shown in Figure 1. The studied preparation of immobilized subtilisins is a pharmaceutical substance of the drug Trombovazim (Producer AO SCPB, Novosibirsk, Russia).

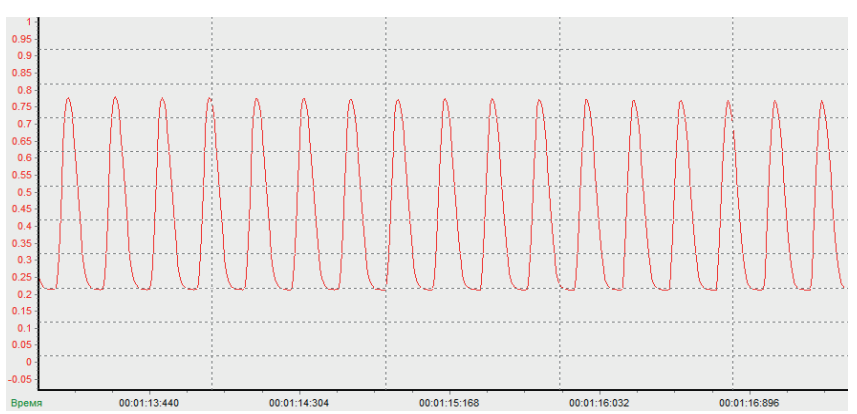

Fig. 1. Record of Isolated Rat Heart Working

The studied component was added to the perfusion solution in 4 concentrations: $150 \mathrm{IU} / \mathrm{L}, 300 \mathrm{IU} / \mathrm{L}, 600 \mathrm{IU} / \mathrm{L}$ and $1200 \mathrm{IU} / \mathrm{L}$. The initial indicators of the functioning rat heart differed among themselves, therefore, the changes under the influence of the investigated component were evaluated in percent in relation to the initial indicators - "Control". The obtained data were subjected by the statistical analysis using the program StatPlus 2009 Professional (USA). The statistical significance of the results was evaluated using Student's t-test for related samples (before and after administration of the test drug) at a significance level of $\mathrm{p}<0.05$.

\section{Results}

A. Study with a dose of $150 \mathrm{IU} / \mathrm{L}$. The heart rate significantly decreased, starting from 20 minutes of perfusion and was $7 \%$ in relation to the initial data. In the presence of subtilisins, the maximum increase of the LVP index was noted at the 20 minutes of perfusion by $32 \%$ compared with the initial values. The evaluation of the heart efficiency (HE) showed a significant increase by $17 \%$ at the 20 minutes of perfusion. The data are summarized in Fig. 2.

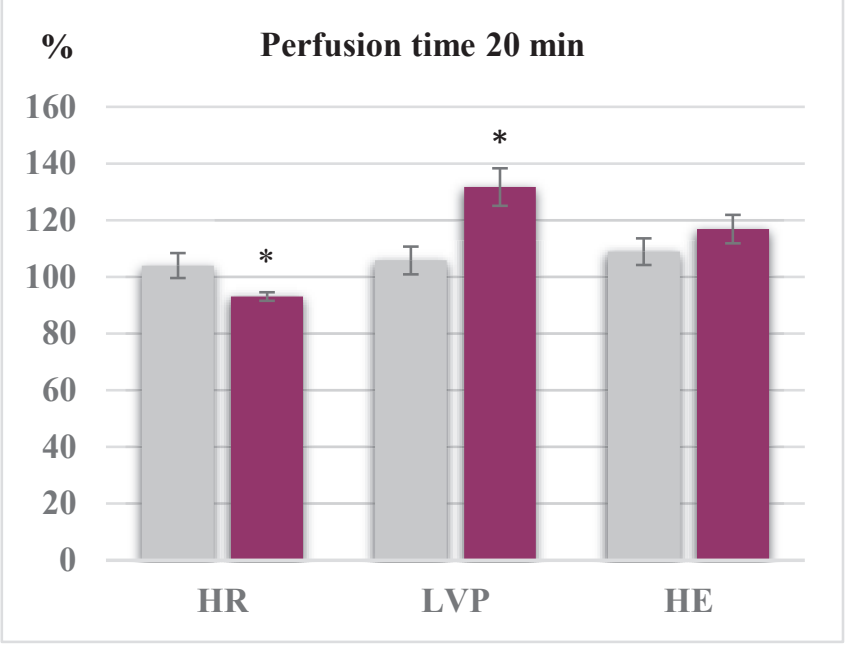

Fig. 2. Perfomance indicators of isolated rat heart, when adding $150 \mathrm{IU} / \mathrm{L}$ of immobilized subtilisins $(\mathrm{M} \pm \mathrm{m}),{ }^{*}-\mathrm{p}<0,05$ against the control.

Note. Grey column - control (Krebs-Henseleit), lilac column - experiment (Immobilized subtilisins); HR - heart rate, LVP - left ventricular pressure, $\mathrm{HE}$ - heart efficiency.

B. Study with a dose of $300 \mathrm{IU} / \mathrm{L}$. The heart rate significantly decreased, starting from 20 minutes of perfusion, and was $5 \%$ in relation to the control. During perfusion with subtilisins the maximum increase of the LVP index was observed at the 20 minutes of perfusion by $36 \%$. The evaluation of the heart efficiency (HE) showed a significant increase by $30 \%$ at the 20 minutes of perfusion. The data are summarized in Fig. 3.

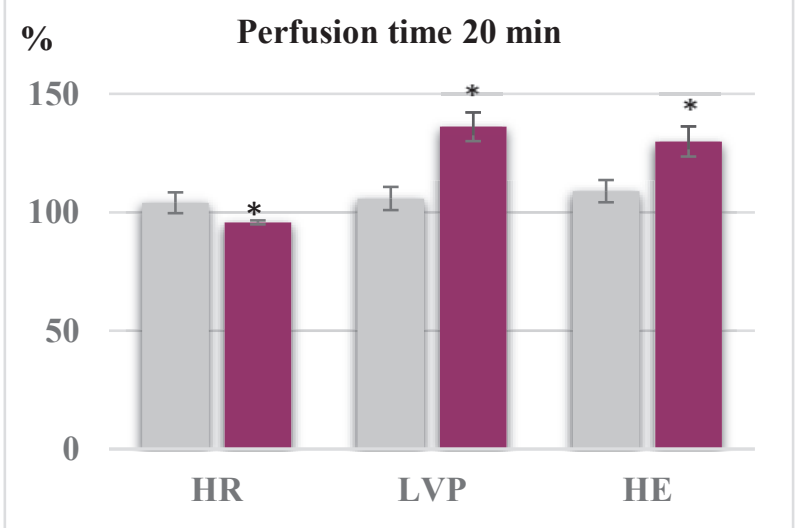

Fig. 3. Perfomance indicators of isolated rat heart, when adding $300 \mathrm{IU} / \mathrm{L}$ of immobilized subtilisins $(\mathrm{M} \pm \mathrm{m}),{ }^{*}-\mathrm{p}<0,05$ against the control.

Note. Grey column - control (Krebs-Henseleit), lilac column - experiment (Immobilized subtilisins); HR - heart rate, LVP - left ventricular pressure, $\mathrm{HE}$ - heart efficiency. 
C. Study with a dose of $600 \mathrm{IU} / \mathrm{L}$. The heart rate significantly decreased by $10 \%$ in relation to the initial data at 20 minutes of perfusion. There was a maximum increase in the rate of LVP index at 20 minutes of perfusion by $22 \%$, compared with the control. The evaluation of the heart efficiency (HE) showed a significant increase by $18 \%$ at the 20 minutes of perfusion. The data are summarized in Fig.4.

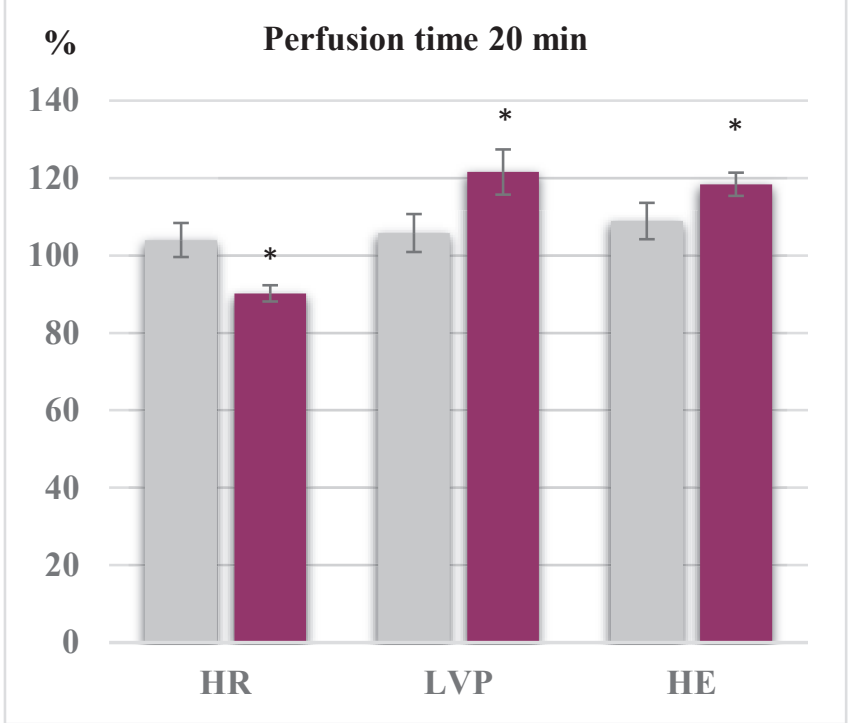

Fig. 4. Perfomance indicators of isolated rat heart, when adding $600 \mathrm{IU} / \mathrm{L}$ of immobilized subtilisins $(\mathrm{M} \pm \mathrm{m}), * \mathrm{p}<0,05$ against the control.

Note. Grey column - control (Krebs-Henseleit), lilac column - experiment (Immobilized subtilisins); HR - heart rate, LVP - left ventricular pressure, $\mathrm{HE}$ - heart efficiency

Study with a dose of $1200 \mathrm{IU} / \mathrm{L}$. The heart rate was $9 \%$ in relation to the control at the 20 minutes of perfusion. The LVP index increased by $25 \%$ at 20 minutes of perfusion and relative to the control. The evaluation of the heart efficiency (HE) showed a significant increase by $20 \%$ at the 20 minutes of perfusion. The data are summarized in Fig. 5.

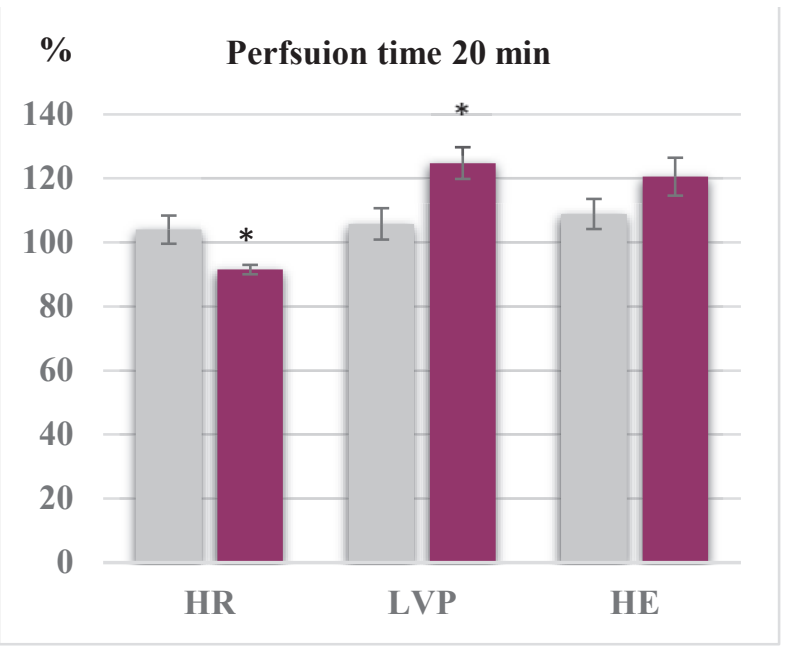

Fig. 5. Perfomance indicators of isolated rat heart, when adding 1200 IU/L of immobilized subtilisins $(\mathrm{M} \pm \mathrm{m}),{ }^{*}-\mathrm{p}<0,05$ against the control. Note. Grey column - control (Krebs-Henseleit), lilac column - experiment (Immobilized subtilisins); HR - heart rate, LVP - left ventricular pressure, $\mathrm{HE}$ - heart efficiency.

\section{Consclusion}

The studies have shown that immobilized subtilisins have a direct effect on the heart muscle. The immobilized subtilisins increase the force contraction of the left ventricle and reduce the heart rate. There are observed positive inotropic and negative chronotropic effects with perfusion of immobilized subtilisins, most pronounced at a dose of $300 \mathrm{IU} / \mathrm{L}$ These circumstances have a scientific and practical interest, since a combination of positive inotropic and negative chronotropic effects establishes a more economical mode of heart function. The immobilized subtilisins have great prospects using in cardiology.

\section{References}

[1] P.G. Madonov, S.V. Mishenina, D.N. Kinsht, N.V. Kikhtenko "Chemical and pharmacological properties of subtilisins," Sibirskij nauchnyj medicinskij zhurnal, vol. 36 (3), pp. 13-22, 2016.

[2] P.G. Madonov, D.N. Kinsht, N.V. Kikhtenko, S.V. Mishenina "Targeted pharmacodynamics of subtilisins," Sibirskij nauchnyj medicinskij zhurnal, vol. 36 (4), pp. 15-24, 2016.

[3] S.M. Minasyan, M.M. Galagudza, D.L. Sonin "Methods of perfusion of an isolated rat heart," Regionarnoe krovoobrashchenie i mikrocirkulyaciya, vol. 8(4), pp. 54-59, 2009.

[4] R.G. Merin "The isolated heart preparation," British Journal of Anesthesia, vol. 60(8), suppl. 1, pp. 28-34. 1988. https://doi.org/10.1093/bja/60.suppl 1.28s.

[5] F.J. Sutherland, K.E. Baker, D.J. Hearse, M.J. Shattock "Mouse isolated perfused heart: characteristics and cautions," Clinical and Experimental Pharmacology, vol. 30(11), pp. 867-878, 2003. https://doi.org/10.1046/j.1440-1681.2003.03925.x.

[6] R.A. Knyazev, N.V. Trifonova, A.R. Kolpakov, L.M. Polyakov "Investigation of cardiotoxic effect of A-1 apo + D actinomycin complex on isolated rat heart," Patologiya krovoobrashcheniya i kardiokhirurgiya, vol.20 (4), pp. 88-95. 2016. http://doi.org/10.216881681- 3472-2016-4-88-95 